\title{
The Effects of Transcranial Direct Current Stimulation on Mental Health of Veterans with Psychiatric Disorders
}

\section{Shohreh Vafaye Sisakht, Khosro Ramezani*}

Department of Psychology, Yasooj Branch, Islamic Azad University, Yasooj, Iran

\section{ABSTRACT}

Introduction: Using direct electrical stimulation of is a therapeutic technique that directly affects the spontaneous activity in the cortical regions. The aim of this study is to determine the effects of direct stimulation of the brain lining the skull (TDCS) on improving the mental health of veterans with psychiatric disorders. Materials and Methods: This study was quasi-experimental investigation with pre-test and post-test computations. Sixteen veterans with psychiatric problems from Yasouj city were assigned randomly to experimental and control groups. The experimental group received 10 sessions of treatment. The SCL-90 questionnaire was used to collect data. All participants took part in the pre-test and post-test. Results: The results showed that the treatment with TDCS had significant therapeutic effects on mental health. Conclusion: The results demonstrated the effectiveness of treatment of TDCS on the mental health of veterans with psychiatric disorders.

Key words:

1. Brain

2. Mental Health

3. Skull

*Corresponding Author: Khosro Ramezani

E-mail: Kh.ramezani@iauyasooj.ac.ir 
اثرات تحريك الكتريكى مستقيم مغز از وراى جمجمه بر سلامت روان جانبازان با اختلالات روانى

$$
\text { كروه روانشناسى، واحد ياسوج، دانشَاه آزاد اسلامى، ياسوج، ايران خسرو رمضانى" }
$$

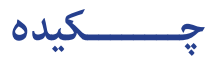

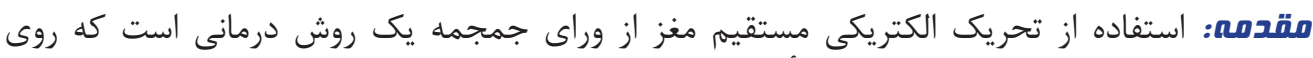

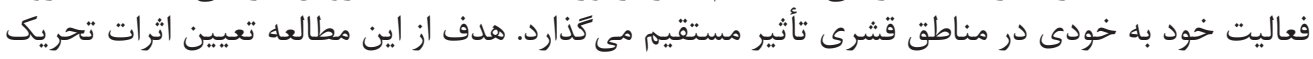

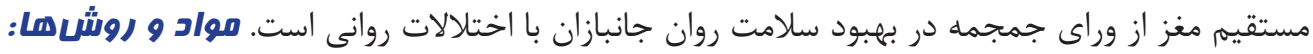

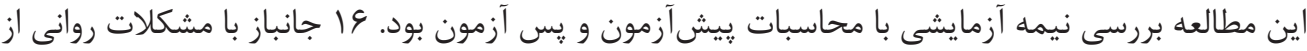

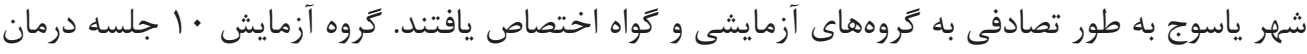

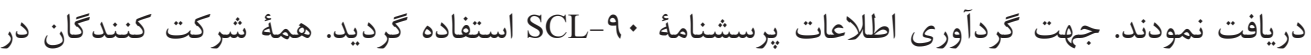

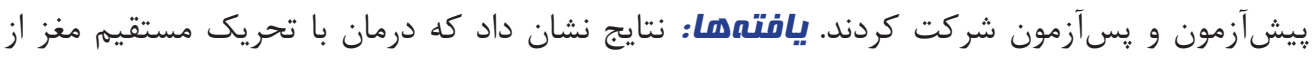

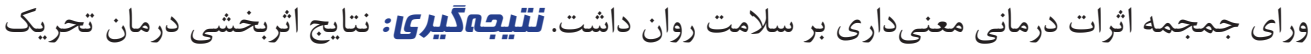

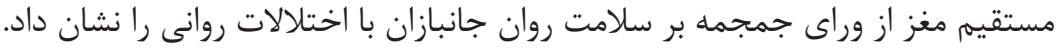

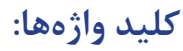

1 1

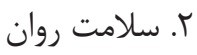
r. جمجمه

$$
\text { " نويسنده مسئول: خسرو رمضانى }
$$

آدرس الكترونيكى: Kh.ramezani@iauyasooj.ac.ir 


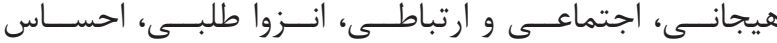

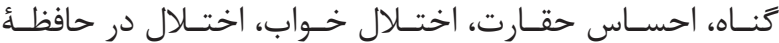

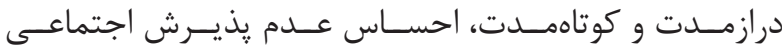

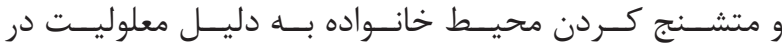

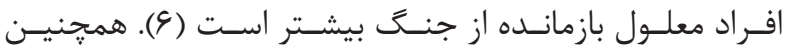

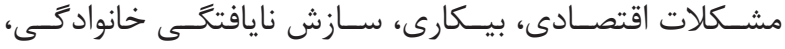

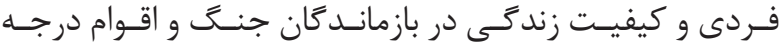

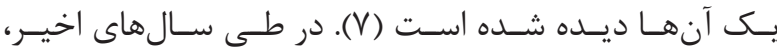

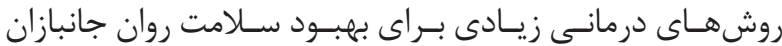

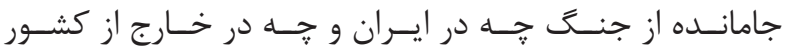

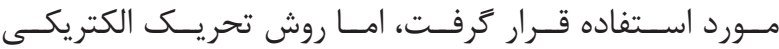

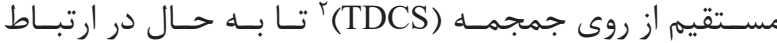

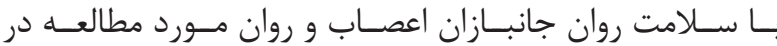

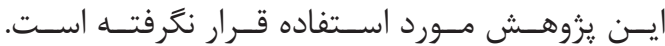

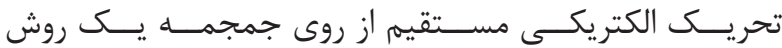

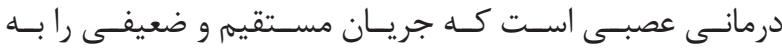

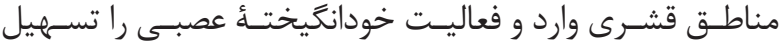

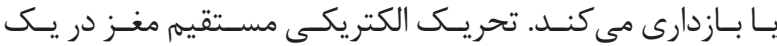

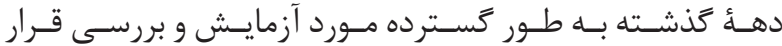

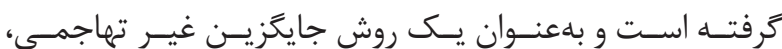

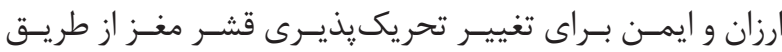

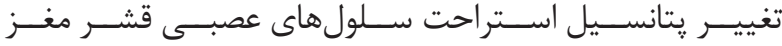

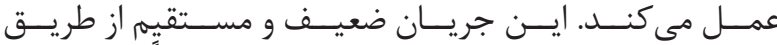

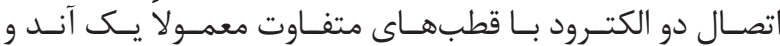

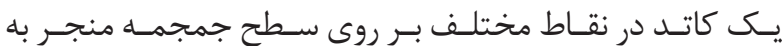

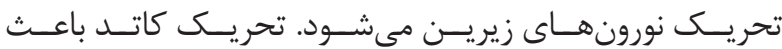

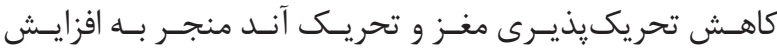

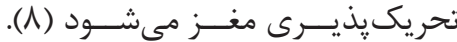

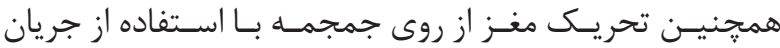

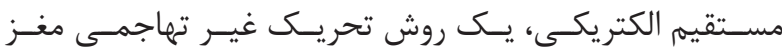

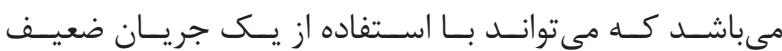

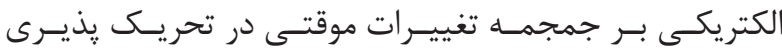

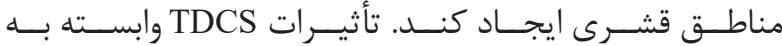

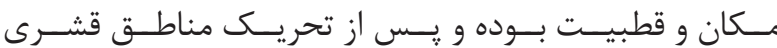

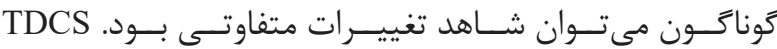

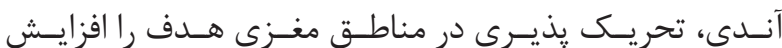

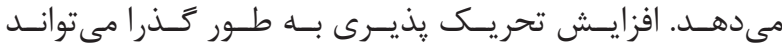

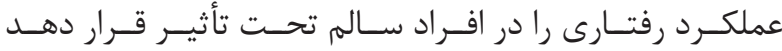

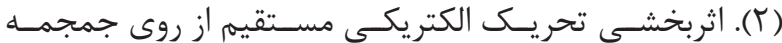

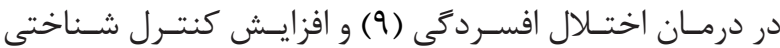

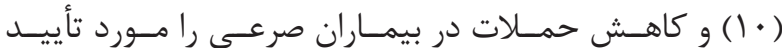

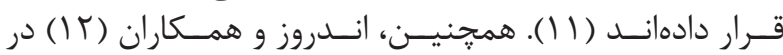

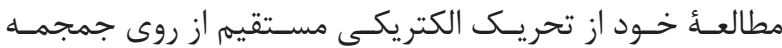

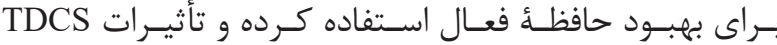

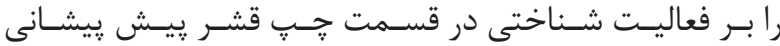

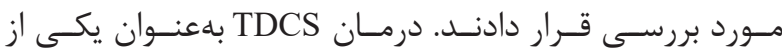

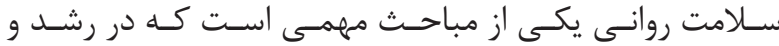

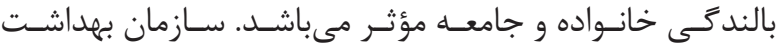

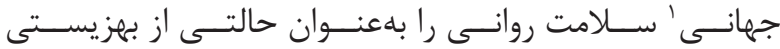

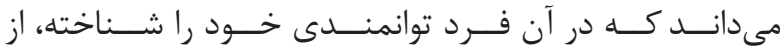

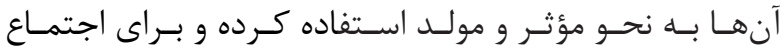

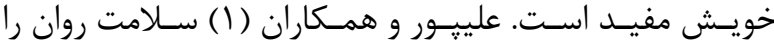

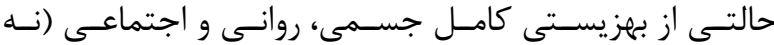

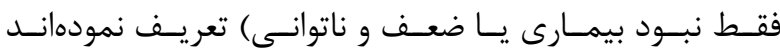

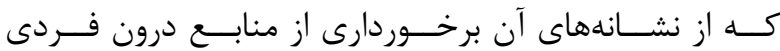

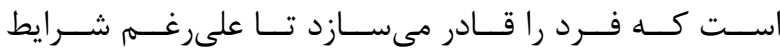

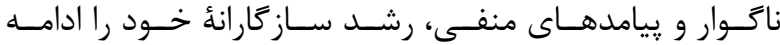

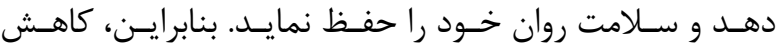

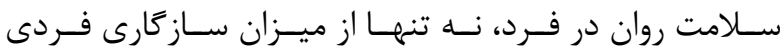

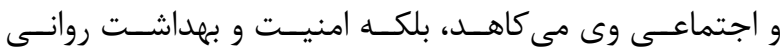

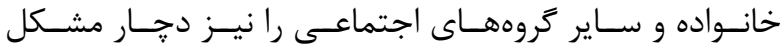

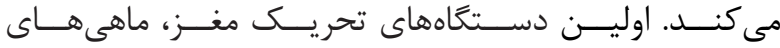

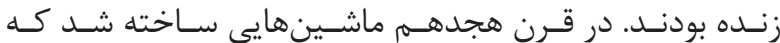

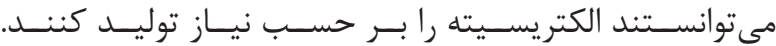

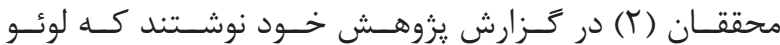

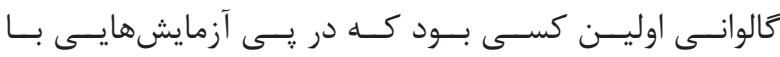

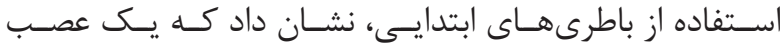

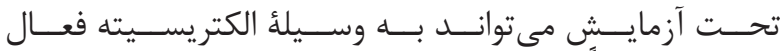

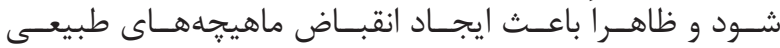

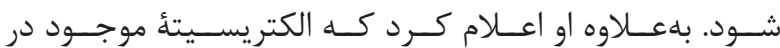

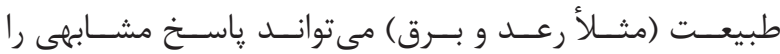

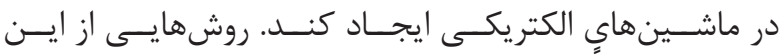

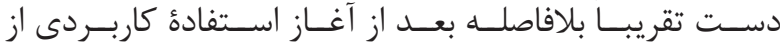

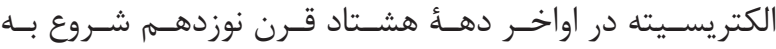

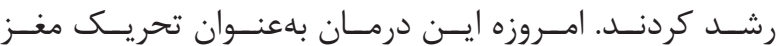

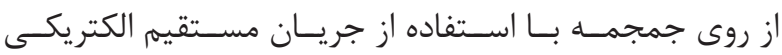

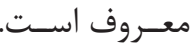

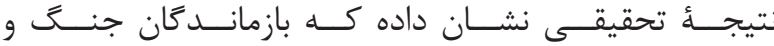

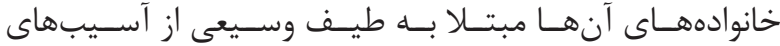

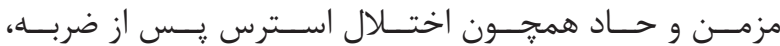

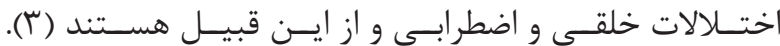

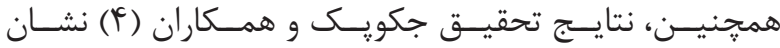

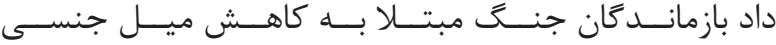

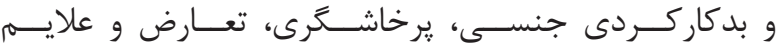

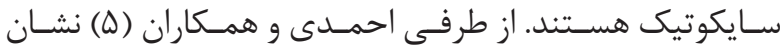

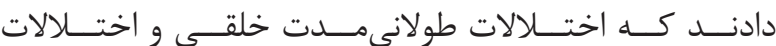

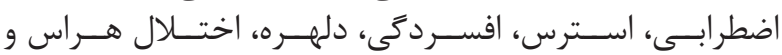

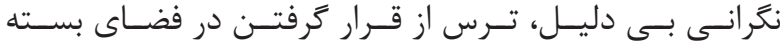

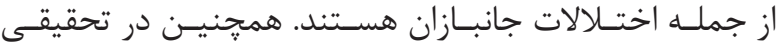

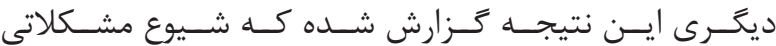

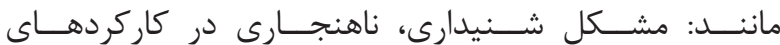

${ }^{1}$ World health organisation

${ }^{2}$ Transcranial direct current stimulation 


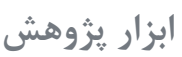

\section{ير سشنامة سلامت روان SCL-90-R}

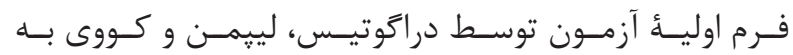

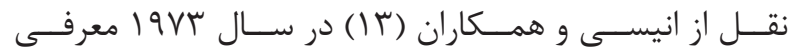

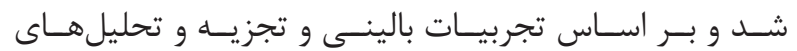

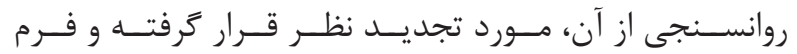

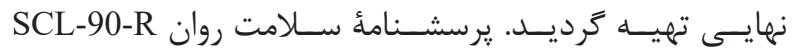

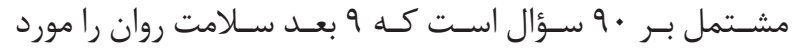

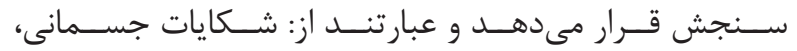

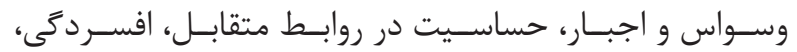

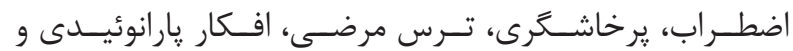

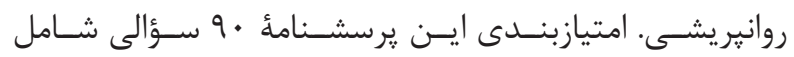

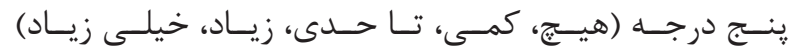

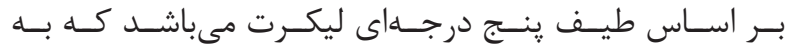

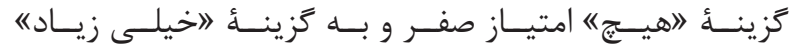

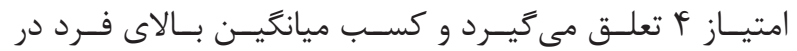

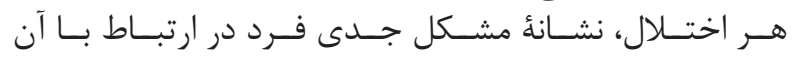

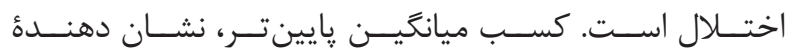

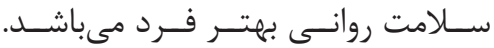

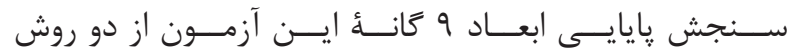

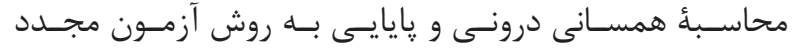

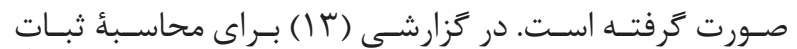

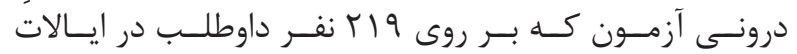

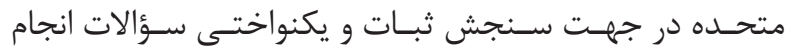

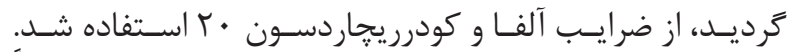

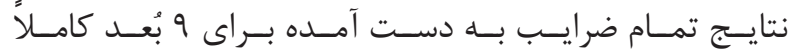

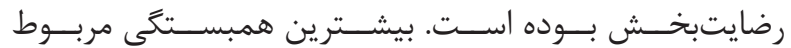

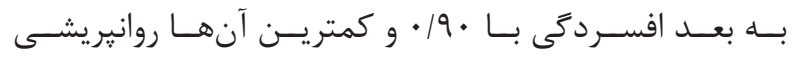

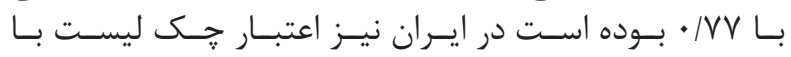

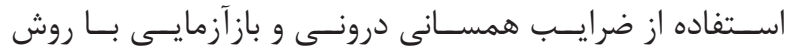

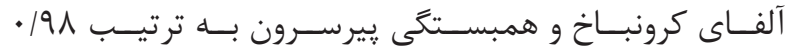

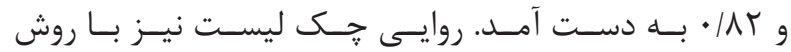

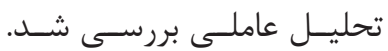

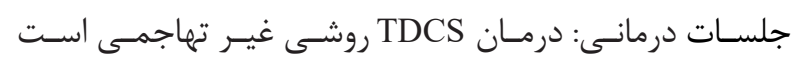

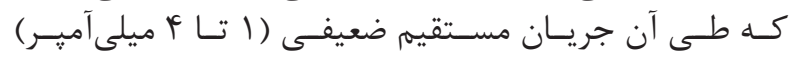

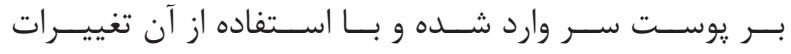

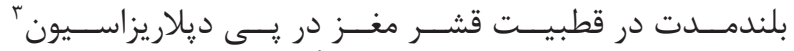

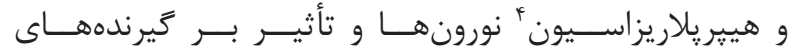

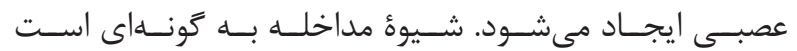

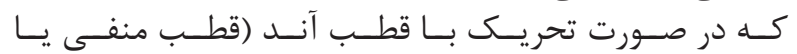

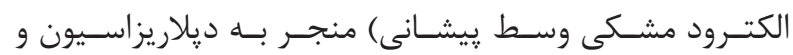

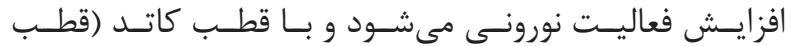

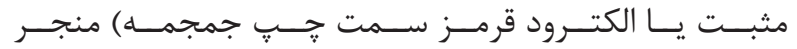

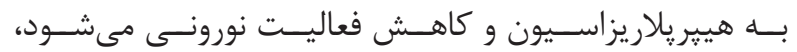

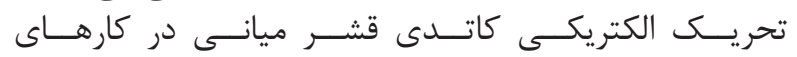

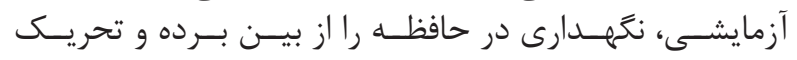

${ }^{3}$ Depolarization

${ }^{4}$ Hyperpolarization

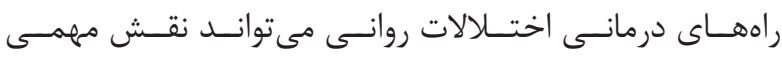

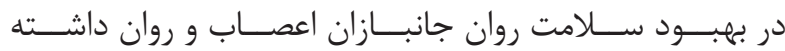

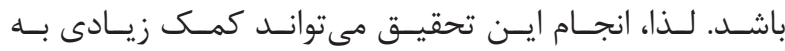

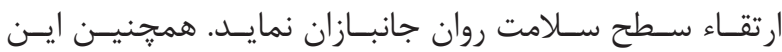

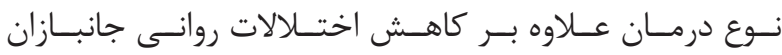

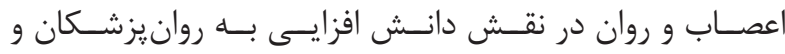

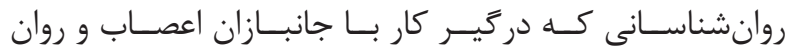

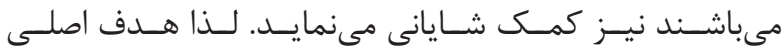

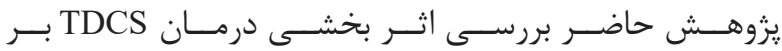

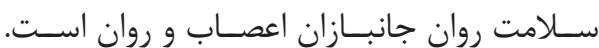

مواد و روشها

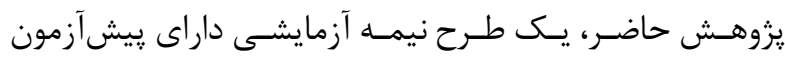

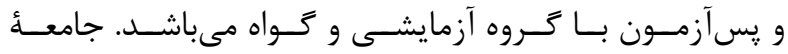

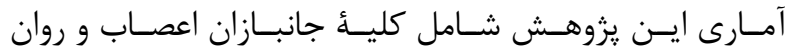

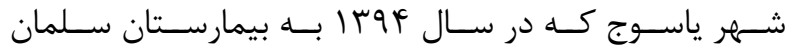

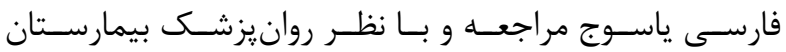

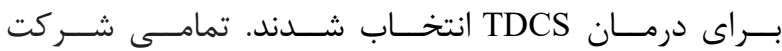

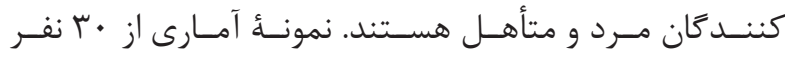

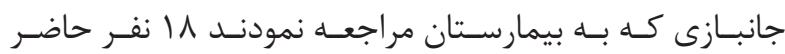

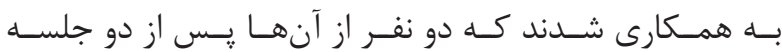

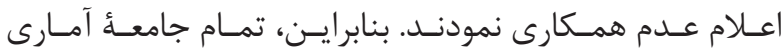

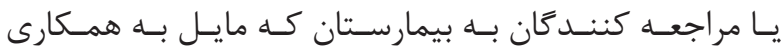

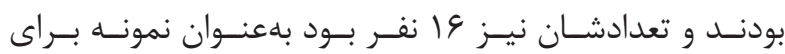

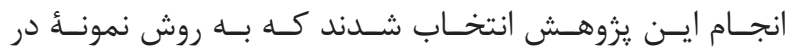

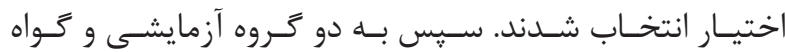

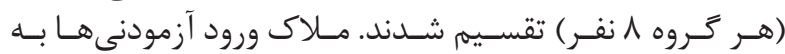

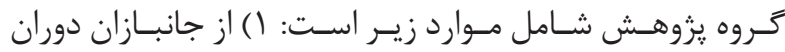

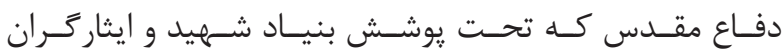

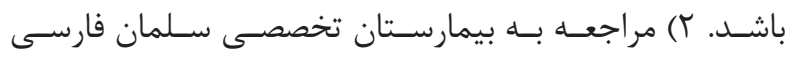

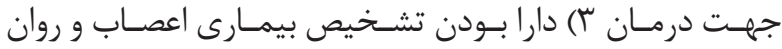

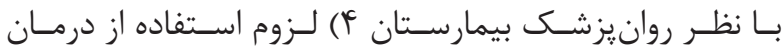

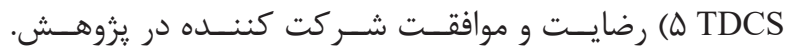

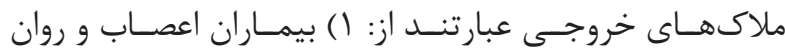

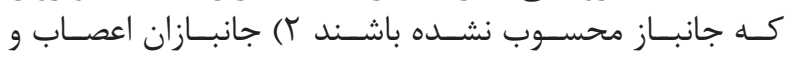

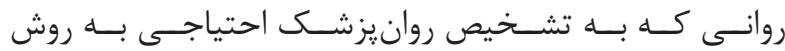

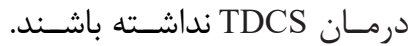

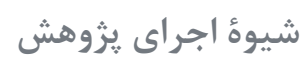

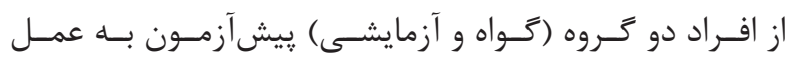

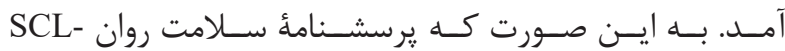

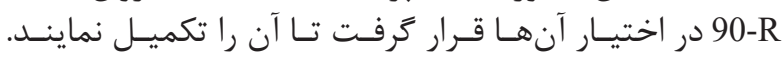

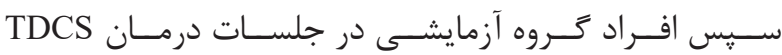

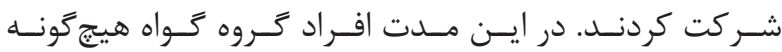

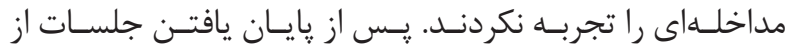

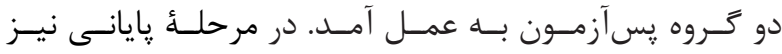

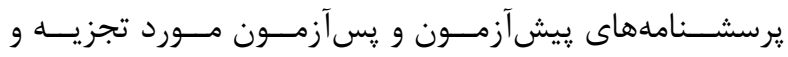

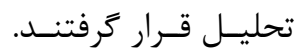


يافتهها

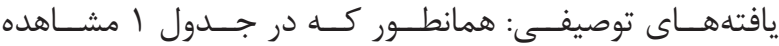

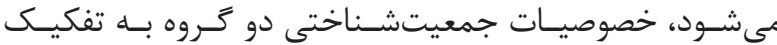

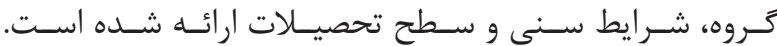

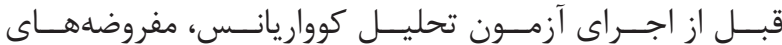

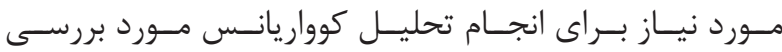

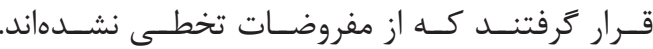

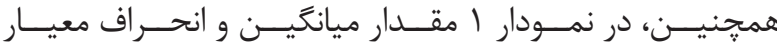

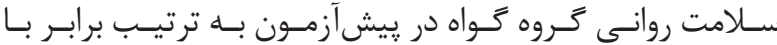

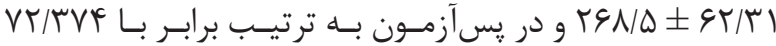

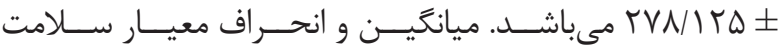

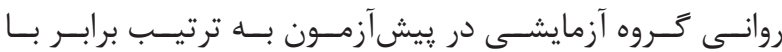
روأس I AF/AVD I Fr/GYq TDCS

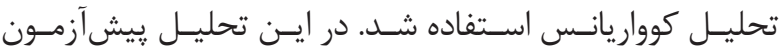

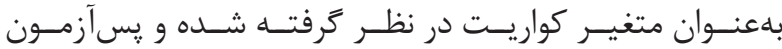

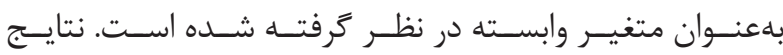

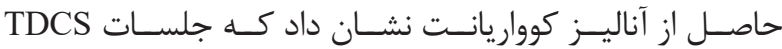

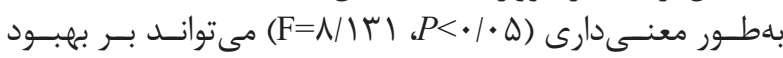

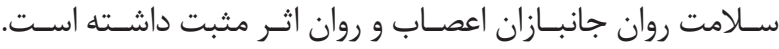

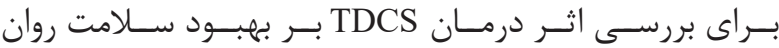

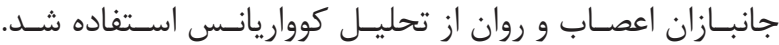

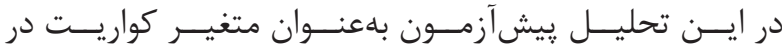

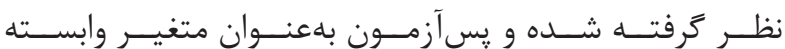

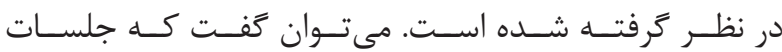

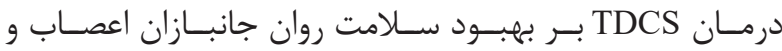

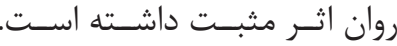

آنــدى، تحكيــم حافظـــهـ را ســـرعت مىبخشــد.

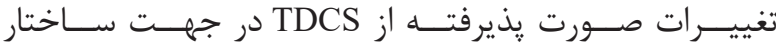

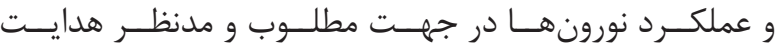

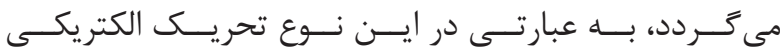

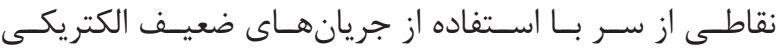

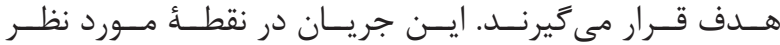

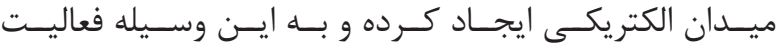

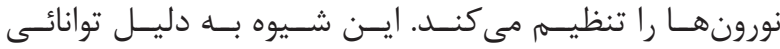

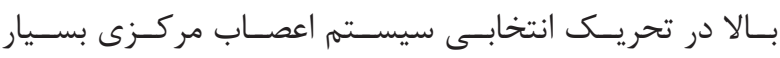

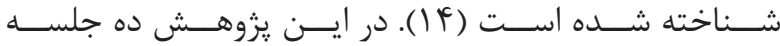

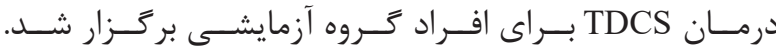

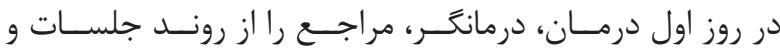

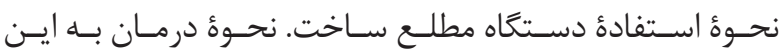

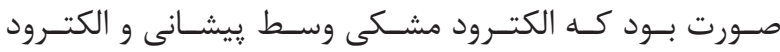

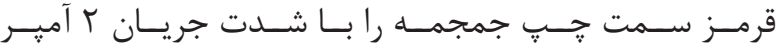

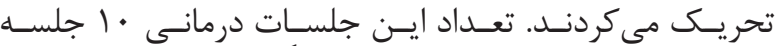

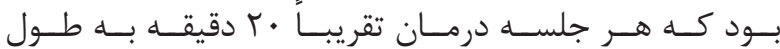

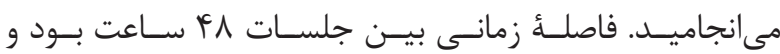

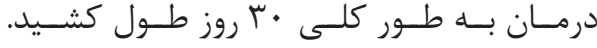
تجزيه و تحليل دادهها

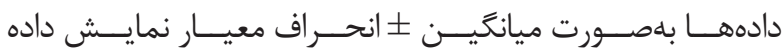

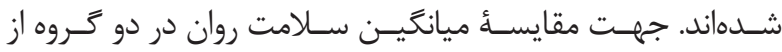

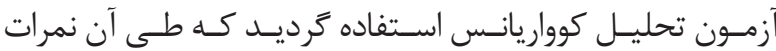

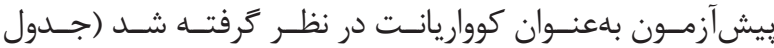

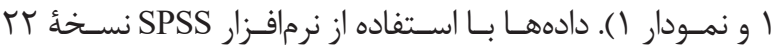

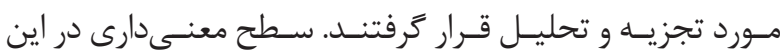

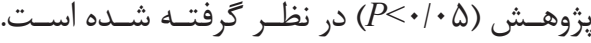

جدول ا- شرايط سنى و ميزان تحصيلات شركت كنندكان در يُروش.

\begin{tabular}{|c|c|c|c|c|}
\hline درصد & تعداد & تحصيلات & سن & كروه \\
\hline ro & r & كمتتر از ديبلهم & \multirow{3}{*}{ هr تا • ه سال } & \multirow{6}{*}{ كَروه آزمايشى } \\
\hline $1 T / \Delta$ & 1 & فوق ديبلم & & \\
\hline ro & r & ليسانسى & & \\
\hline ro & r & كمتر از ديبلمم & \multirow{2}{*}{ بيشتر از •ه سال } & \\
\hline $1 \pi / \Delta$ & 1 & فوق ليسانسى & & \\
\hline $1 \ldots$ & $\Lambda$ & \multicolumn{2}{|c|}{ جمع } & \\
\hline ro & $r$ & كمتر از ديبلمم & \multirow{2}{*}{ هr تا • ه سال } & \multirow{7}{*}{ كروه كواه } \\
\hline $1 \pi / \Delta$ & 1 & ليسـانسى & & \\
\hline $\mid r / \Delta$ & 1 & كمتتر از ديبلمم & \multirow{4}{*}{ بيشتر از •ه سال } & \\
\hline ro & r & ديبلمم & & \\
\hline $1 \pi / \Delta$ & 1 & فوق ديبلمم & & \\
\hline $1 \pi / \Delta$ & 1 & ليسانسى & & \\
\hline $1 \ldots$ & $\lambda$ & \multicolumn{2}{|c|}{ جمع } & \\
\hline- & 18 & \multicolumn{3}{|c|}{ جمع كل } \\
\hline
\end{tabular}




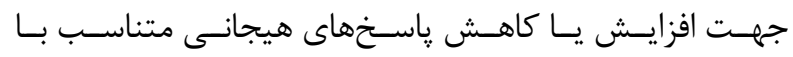

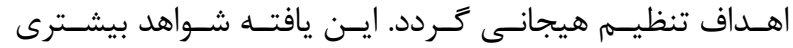

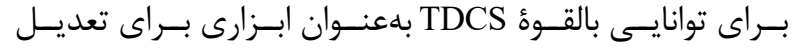

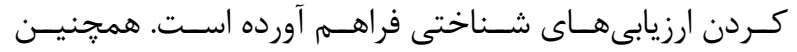

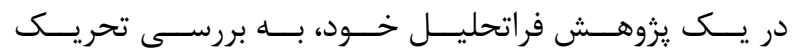

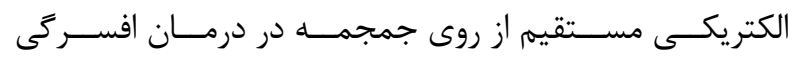

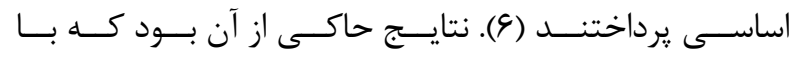

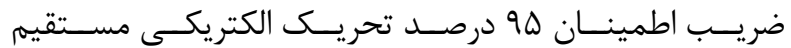

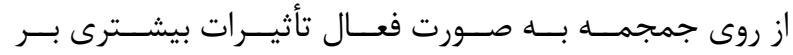

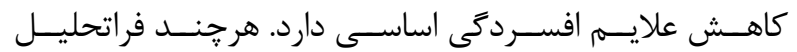

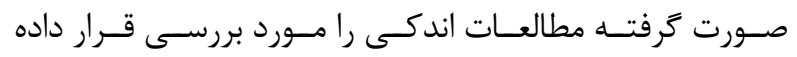

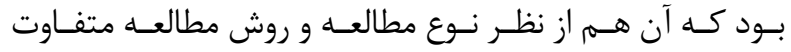

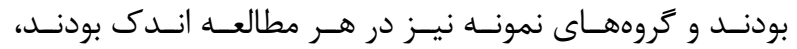

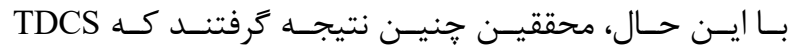

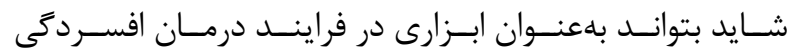

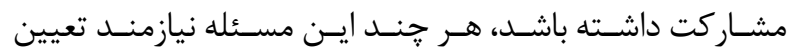

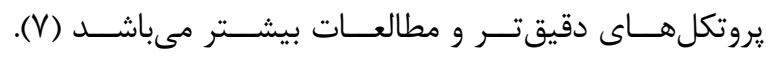

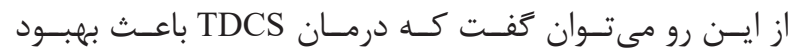

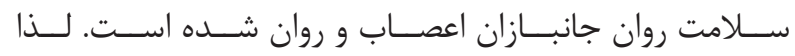

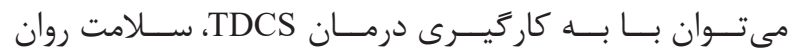

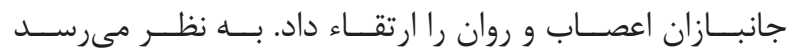

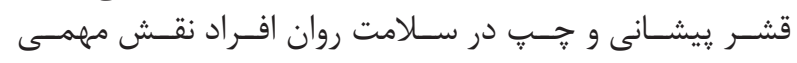

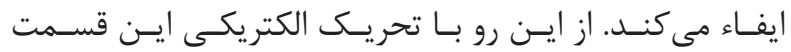

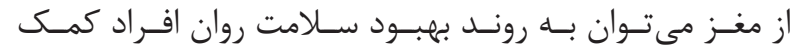

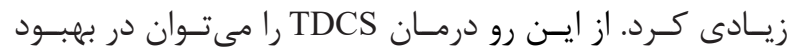

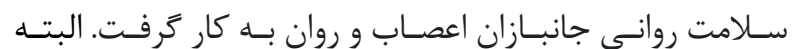

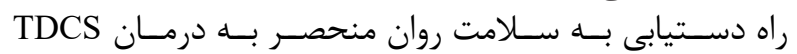

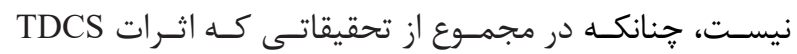

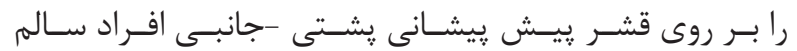

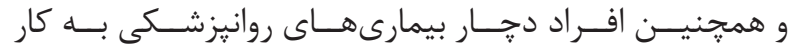

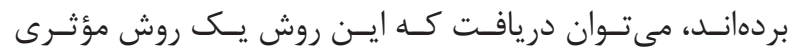

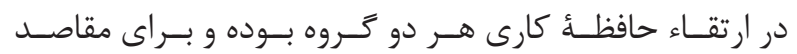

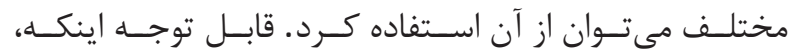

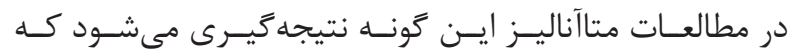

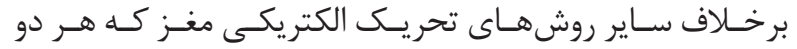

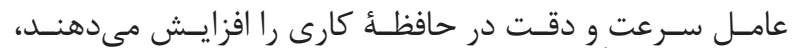

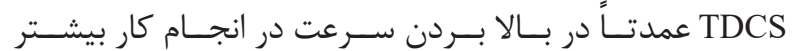

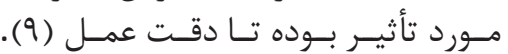

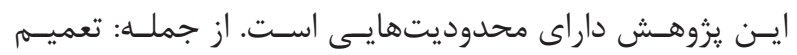

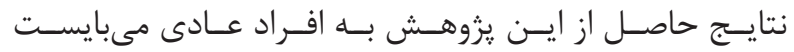

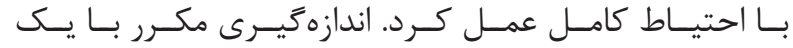

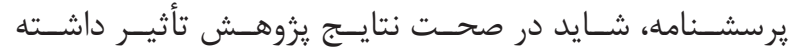

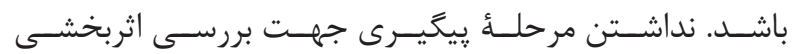

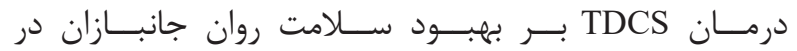

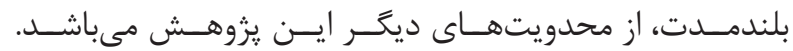

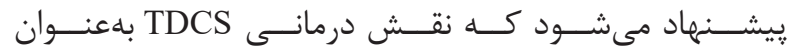

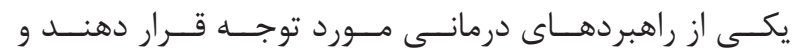

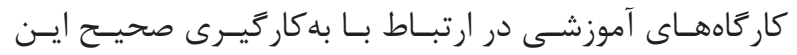

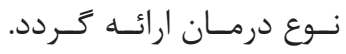

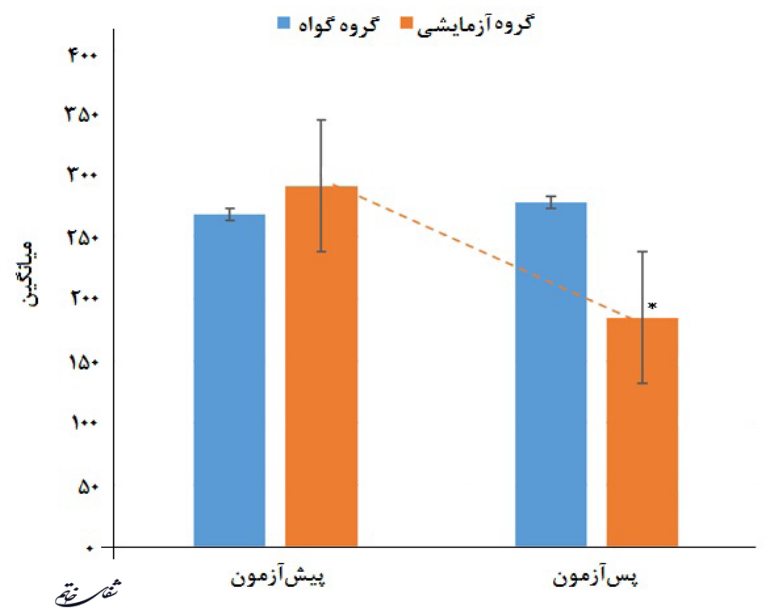

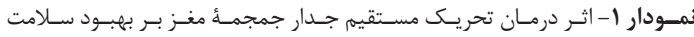

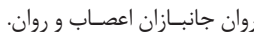

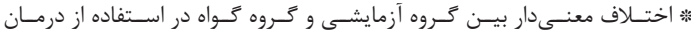

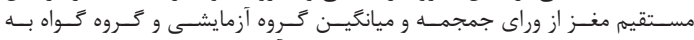

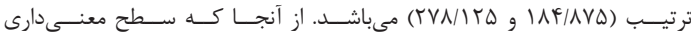

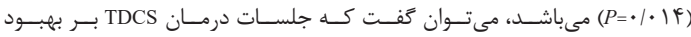

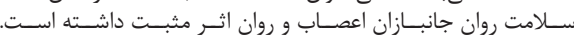

بحث و نتيجه كيرى

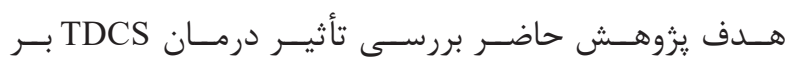

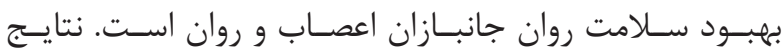

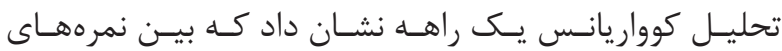

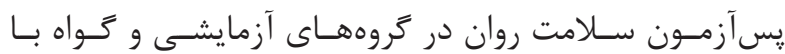

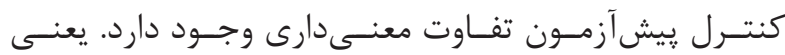

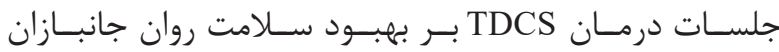

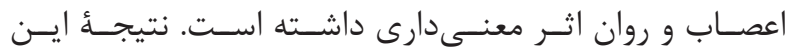

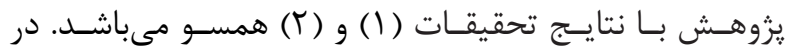

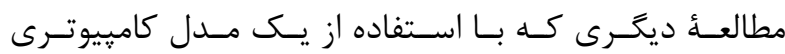

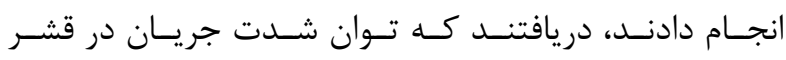

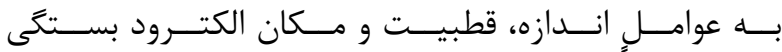

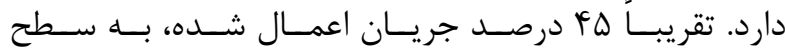

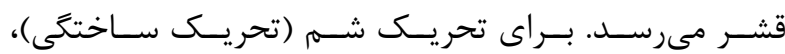

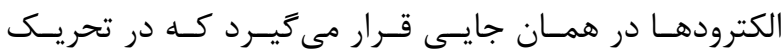

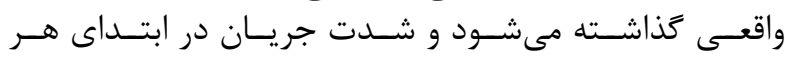

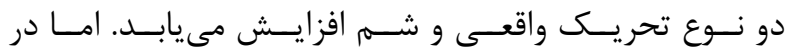

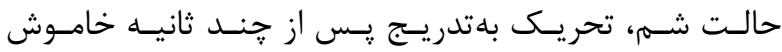

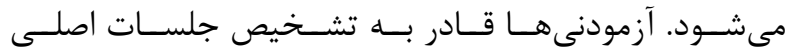

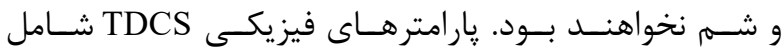

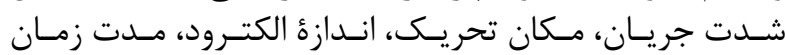

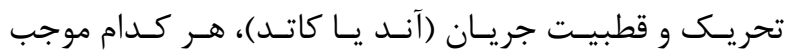

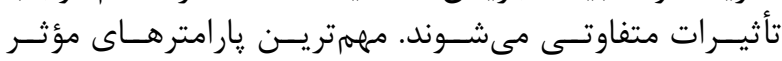

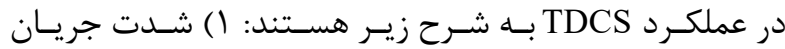

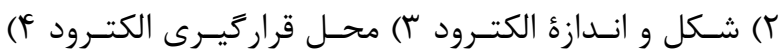

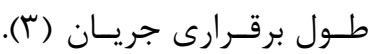

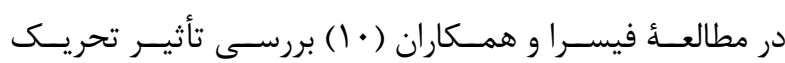

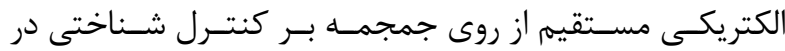

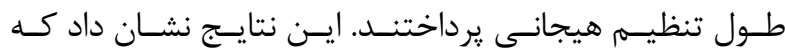

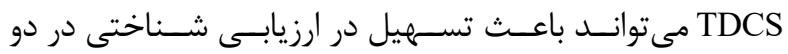




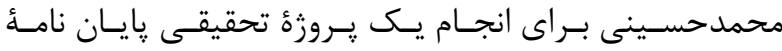

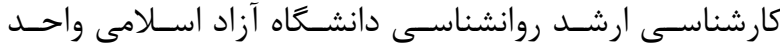

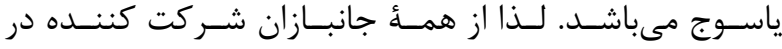

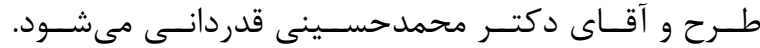

1. Alipor A, Aliakbari Dehkordi M, Hassanzadeh Pashang S, Faraji F. The effect of emotional intelligence training on mental health of the patients hospitalized in cardiology department of shahid modares hospital Tehran, Iran. Shefaye Khatam. 2016; (4)3: 41-50.

2. Fregni F, Boggio PS, Nitsche MA, Marcolin MA, Rigonatti SP, Pascual-Leone A. Treatment of major depression with transcranial direct current stimulation. Bipolar Disorder. 2006; 8(2): 203-4.

3. Grieger TA, Cozza SJ, Ursano RJ, Hoge C, Martinez PE, Engel CC, et al. Posttraumatic stress disorder and depression in battle-injured soldiers. Am J Psychiatry. 2006; 163(10): 1777-83.

4. Jakupcak M, Tull MT, McDermott MJ, Kaysen D, Hunt S, Simpson T. PTSD symptom clusters in relationship to alcohol misuse among Iraq and Afghanistan war veterans seeking post-deployment VA health care. Add Behav. 2010; 35(9): 840-3.

5. Ahmadi K, Reshadatjoo M, Sepehrvand N, Ahmadi $\mathrm{P}$, Yaribeygi H. Evaluation of vicarious PTSD among children of Sardasht chemical warfare survivors 20 years after Iran-Iraq war. J Appl Sci. 2010; 10(23): 3111-6.

6. Mulrow CD, Aguilar C, Endicott JE, Velez R, Tuley MR, Charlip WS, et al. Association between hearing impairment and the quality of life elderly in diviuals. $\mathrm{J}$ Am Geri Soc. 1990; 38(1): 45-50.

7. Williams RM, Turner AP, Hatzakis JM, Bowen JD, Rodriquez AA, Haselkorn JK. Prevalence and correlates of depression among veterans with multiple sclerosis. Neurology. 2005; 64(1): 75-80.

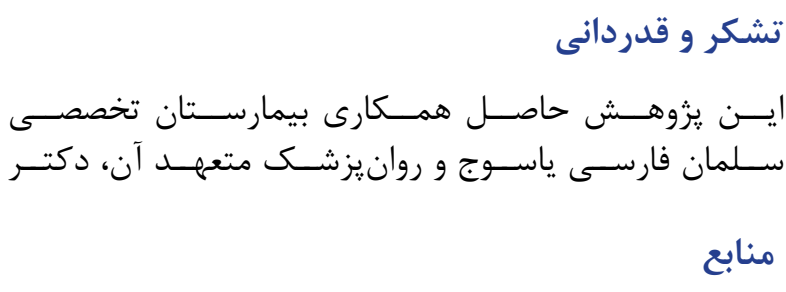

8. Brunoni AR, Nitsche MA, Blognini N, Bikson M, Wagner $\mathrm{T}$, Merabet $\mathrm{L}$, et al. Clinical research with transcranial direct current stimulation (TDCS): challenges and future directions. Brain Stimul. 2012; 5(3): 175-95.

9. Brunoni AR, Ferrucci R, Bortolomasi M, Vergari M, Tadini L, Boggio PS, et al. Transcranial direct current stimulation (tDCS) in unipolar vs. bipolar depressive disorder. Prog Neuropsychopharmacol Biol Psychiatry. 2011; 35(1): 96-101.

10. Feesera M, Prehn K, Kazzer P, Mungee A, Bajbouj M. Transcranial direct current stimulation enhances cognitive control during emotion regulation. Brain Stimul. 2014; 7(1): 105-12.

11. San-juan D, Morales-Quezada L, Garduño AJ, Alonso-Vanegas M, Fernández MF, López DA, et al. Transcranial direct current stimulation in epilepsy. Brain Stimul. 2015; 8(3): 455-64.

12. Andrews SC, Hoy KE, Enticott PG, Daskalakis ZJ, Fitzgerald PB. Improving working memory: the effect of combining cognitive activity and anodal transcranial direct current stimulation to the left dorsolateral prefrontal cortex. Brain Stimulation. 2011; 4: 84-9.

13. Anisi. J. Akbar. Fatma, Majdi. Muhammad, Atashkar. Majid, the victim. Venus (1390). Czech normalization list of symptoms of mental disorders staff of the armed forces. Military Psychology Quarterly. 2012; 2(5). 37-29.

14. Nitsche MA, Boggio PS, Fregni F, Pascual-Leone A. Treatment of depression with transcranial direct current stimulation (tDCS): a review. Exprimental Neurologh. 2009; 219: 14-9. 